\title{
An Agent-Based Model of Santa Cruz Island Foxes (Urocyon littoralis santacruzae) which Exhibits an Allee Effect
}

\author{
Shelby M. Scott ${ }^{1}$, Erin N. Bodine ${ }^{1, *}$, Anne Yust ${ }^{2}$
}

\begin{abstract}
In the early 1990s, the island of Santa Cruz off the coast of California experienced a rapid decline of the canid Urocyon littoralis santacruzae. The reasons behind this decline included disease, predation, competition with other species, and genetic bottlenecking. We present an individual-based model that simulates the population of Santa Cruz island foxes. Through our model we provide evidence of the Allee effect in the Santa Cruz island fox population which is exacerbated by selection for reduced reproductive probability.
\end{abstract}

Keywords: Santa Cruz island fox, agent-based model, Allee effect

\section{Introduction}

The California Channel Islands are home to many species, all which co-exist within the varied vegetation present on these islands. One of these species is a small, territorial, monogamous canid known as the island fox (Urocyon littoralis). The island fox is a derivative of the mainland grey fox, capable of surviving and making their territories in any environment present on the islands [8]. During mating season (late January to March), monogamous mating pairs are formed between individuals in overlapping territories. These monogamous pairings are maintained until one member of the pair dies. Once a female fox is impregnated, her gestation period lasts for 50-53 days at which point she births between 1 and 3 pups. These pups remain in their natal territory for the first year of their lives before searching for their own mates and territories [9].

Six of the eight California Channel Islands support island fox populations (San Clemente, San Nicolas, San Miguel, Santa Catalina, Santa Cruz, and Santa Rosa). In the early 1990s, conservationists observed rapid population decline in all of the island fox populations [9]. The reasons for this rapid decline was predation by golden eagles (Aquila chrysaetos), competition with island spotted skunks (Spilogale gracilis amphiala), introduction of canine distemper virus and rabies for which the foxes had no natural immunity, and the potentially decreasing genetic fitness of the population [9]. The specific reason for decline on each island varied.

The island fox population on Santa Cruz (Urocyon littoralis santacruzae) experienced population decline primarily due to predation by golden eagles (Aquila chrysaetos) [7, 9, 11, 19] though disease and resource competition with island spotted skunks (Spilogale gracilis amphiala) may have also played a role $[8,14]$. In 1994 , the size of island fox population

\footnotetext{
${ }^{1}$ Department of Mathematics \& Computer Science, Rhodes College, Memphis, TN

${ }^{2}$ Department of Mathematics, Birmingham-Southern College, Birmingham, AL

* Correspondence: bodinee@rhodes.edu
} 
on Santa Cruz Island was estimated to be 1,465 , but by 2003 that number had declined to between 75 and 100 foxes [9].

There is also evidence of a demographic Allee effect in the island fox populations of Santa Cruz, Santa Rosa, and San Miguel [2]. The Allee effect is seen in populations where a decrease in fitness directly correlates to declining, low population densities $[1,6,15]$. There are two different forms of the Allee effect, a component Allee effect and a demographic Allee effect. A component Allee effect is a positive relationship between any component of individual fitness and population size. Meanwhile, a demographic Allee effect occurs when there is a positive relationship between any component of total (population) fitness and population size. The demographic Allee effect can be observed as a reduction in the per capita growth rate at low population sizes [15]. See Figure 1 for a graphical representation of this.

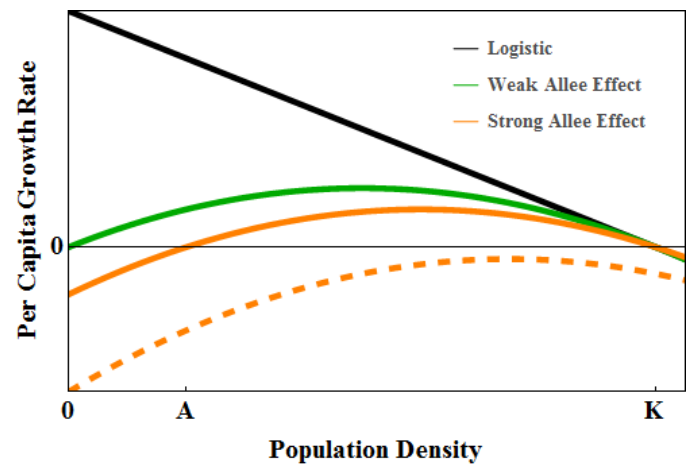

Figure 1: The per capita growth rate of a population with respect to population density in four different scenarios: (1) The population grows logisitically (black), (2) the population experiences a weak Allee effect (green), (3) the population experiences a strong Allee effect but can achieve positive per capita growth rates above a population density $A$ (solid orange), and (4) the population experiences such a strong Allee effect that it never attains positive per capita growth rates. Note $K$ represents carrying capacity.

Most models which incorporate an Allee effect use discrete difference or differential equations in order to quantitatively measure how the per capita growth rates change over time with respect to population size or density (see $[3,4,6,10,16,21]$ for some examples). In 2002, Boukal \& Berec reviewed and classified the variety of existing single-species population dynamic models which incorporated a demographic Allee effect [6]. They found that across all of the models, three possible population outcomes due to the Allee effect occurred. The first is unconditional extinction in which, if the Allee effect is too strong, populations inevitably go extinct, regardless of initial population size (see the dashed orange curve in Figure 1). The second outcome is known as extinction-survival, where two equilibria emerge: $E^{u}$ (an unstable equilibria) and $E^{s}$ (a stable equlibria), where $E^{u}<E^{s}$. Populations that start below $E^{u}$ go extinct, while those starting above $E^{u}$ move towards equilibria at $E^{s}$ (see the solid orange curve in Figure 1 where $E^{u}=A$ and $E^{s}=K$ ). The third outcome is unconditional survival that occurs when all populations, regardless of initial population size, move towards a stable equilibria $E^{s}$ even though the per capita growth rate at low sizes is relatively low (see the green curve in Figure 1 where $E^{s}=K$ ).

In this paper we investigate the effects of decreased genetic fitness on the Santa Cruz island fox population in the absence of other population-reducing factors. Unlike the array of population dynamics models with the Allee effect in the literature, we propose an agentbased model that simulates the dynamics of the Santa Cruz island fox population in order to show that the Allee effect is an emergent property of such a system. Agent-based models (ABMs) are a class of mathematical and computational models in which individuals (or agents) are unique and autonomous entities that can interact with other individuals and also with their environment $[12,13,17,18]$. In Section 2 we present a detailed description of the model using the Overview, Design Concepts, and Details (ODD) Protocol proposed by Grimm, et al. [13, 18]. In Section 3 we present evidence that the Allee effect emerges 
from individual fox behavior and resulting population dynamics both with and without the genetic selection against reproductive success. Finally, in Section 4 we discuss how the model and results presented here can be utilized and expanded upon.

\section{Model}

We created an ABM that simulates the dynamics of the Santa Cruz island fox population over several decades. The model allows for the incorporation a detrimental recessive allele which lowers reproductive success. Here, we describe the ABM using the ODD Protocol as described in $[13,17,18]$.

\subsection{Overview}

Purpose This model simulates the population dynamics and population genetics (of a single gene) of the Santa Cruz island fox population. The model allows for reduced reproductive success in females with a particular recessive allele present in their genome. Using this model we examine whether the Allee effect is an emergent property of the system, and, if so, under what specific conditions.

Entities, State Variables, and Scales Each agent in the model is an individual Santa Cruz island fox. Each fox has state variables for age and the genotype of one gene. Additionally, female foxes have a variable that represents their current gestational week. Each patch within the model defines a fox territory $(0.74 \mathrm{~km} \times 0.74 \mathrm{~km}[9])$ with the total size of the environment being 45 patches $\times 10$ patches, which roughly approximates the size of Santa Cruz Island [5]. These patches are homogeneous without patch-specific variables (this is due to foxes being able to create their territories in any habitat [9]). The model's global parameters include the initial number of female foxes, the initial number of male foxes, the natural death rate of foxes $(\mu)$, the rate of successful reproduction $(r)$, the proportion decrease in fecundity due to a homozygous recessive genotype $(s)$, and the maximum successful reproductive age of the foxes $(a)$. Additionally, $h$ is the fraction of $s$ such that $h s$ represents the proportion decrease in reproductive success due to a heterozygous genotype. A single time step in the system represents one week. We run each simulation for 100 years or until the population becomes extinct.

Process Overview The model is initialized with a user-defined number of male and female foxes. Each male and female is assigned a random age below 312 weeks (about 6 years), the average life span of the island fox [9], and distributed semi-randomly across territories on the island (see Initialization in Section 2.3 for details). During a single time step, individuals with age greater than 40 weeks (about 10 months, reproductive age [9]) disperse throughout the island, moving towards individuals that are potential mates. If a suitable mate is within a $2.96 \mathrm{~km}$ radius (the length of four patches), a mating pair is created. Once the mated pair is established, that pair will attempt to reproduce during the breeding season (weeks 7-11, with week 1 as the first week of January). Mate pairings last for life, and as long as both foxes in a mated pair are alive and younger than the maximum reproductive age $(a)$ they will attempt to reproduce during each breeding season. If mating is successful, the female's gestational period counter is set to one, indicating a pregnancy. The gestational period counter is then incremented by one each time step (week) until it reaches 7. If mating is unsuccessful, the females gestation period will remain at zero. After the 7 -week gestation period is completed, female will birth between 1 and 3 pups. These pups will be assigned a genotype based on the parental genotypes. Lastly, within each time step, foxes die off according to the natural death rate, and each individual ages by one week. 


\subsection{Design Concepts}

Basic Principles The basic principle of this simulation is to demonstrate realistic Santa Cruz island fox population dynamics in which the presence of a detrimental recessive allele decreases reproductive success. We attempt to observe the Allee effect as an emergent property of the system under various levels of percentage decrease in reproductive success due to a homozygous recessive genotype and heterozygous genotype. We let $s \in[0,1]$ represent the proportion decrease in reproductive success due to a homozygous recessive genotype, and let $h \in[0,1]$ represent a proportion of $s$ so that $h s$ represents the proportion decrease in reproductive success due to a heterozygous genotype. This is based on genetic bottlenecking that has been observed in many isolated populations.

Emergence When $s=0$ and $h=0$ (no decrease in reproductive success), and $\mu=0.009$ (natural death rate) the model exhibits logistic growth reaching carrying capacity after about 70 to 110 years, depending on the initial number of individuals in the population. Note, if the natural death rate is increased, the population declines towards extinction. As the value of $s$ and $h$ are increased, the population takes longer to reach carrying capacity, and is more likely to go extinct before 100 years. Additionally, as $s$ and $h$ are increased, the frequency of the recessive allele decreases and approaches 0 over time. The Allee effect appears as an emergent property of the model (with and without genetically linked decreases in reproductive success) and is manifest as an increased probability of having a negative yearly per capita growth rate at low population sizes.

Adaptation As foxes search for mates, the patches around them are defined as possible destinations and preferred destinations. Possible destinations are patches within a radius of $2.96 \mathrm{~km}$ (4 patch lengths) that do not have any other foxes there of the same sex. Preferred destinations are patches within a radius $2.96 \mathrm{~km}$ that include individuals of the opposite sex above the reproductive age that are currently not part of a mated pair. If a preferred destination is unavailable, foxes will move to a possible destination. If there are no possible or preferred destinations available, the foxes will remain on the patch they currently occupy until preferred or possible destination is available.

Objectives The only objectives of the foxes are to find a mate and reproduce. Their movement decisions are based on a preference for finding a suitable mate. However, the criteria for a suitable mate is that they are over reproductive age ( $>40$ weeks) and they currently do not have a mate. The genotype of possible mates are not considered in criteria for a suitable mate.

Learning The foxes have no learning capacity in this model.

Prediction The foxes have no predictive capacity in this model.

Sensing Foxes can sense the age, gender, and mated-status of the foxes within a $2.96 \mathrm{~km}$ radius (4 patch lengths) of themselves in order to determine proper movement and mating. Also, individuals sense whether breeding season is occurring.

Interaction There are interactions between male and female foxes in establishing a mating pair and a territory within the same patch (see the Dispersal submodel in Section 2.3 for details). Additionally, direct interaction occurs within mated pairs during the breeding season (see the Breeding Season submodel in Section 2.3 for details).

Stochasticity Within this system, there are many processes that depend on randomly generated probabilities. Each mated pair's attempt at reproducing is successful with a probability of $r$ for females with a homozygous dominant genotype, $r(1-s)$ for females with a homozygous recessive genotype, $r(1-h s)$ for females with a heterozygous genotype. The genotypes of the initial population are assigned randomly, and the genotypes of offspring are determined by standard Mendelian genetics (see the Set Alleles submodel in Section 2.3 for details). The number of offspring born to any pregnant female is stochastic with a $55.8 \%$ chance of producing 1 offspring, a 35.6\% chance of producing 2 offspring, and an $8.6 \%$ change of producing 3 offspring [9]. The sex of each new offspring is randomly chosen with 
equal probability of being male or being female. Individuals die at each time step with a probability of $\mu$.

Collectives The collectives of this model include mated pairs within their territories. Once offspring are generated, the pups remain on their natal territory until they are able to establish their own territory. If population densities are high, it is possible some offspring stay in their natal territories their entire lives. In this situation, there are individuals in the population who may never have the opportunity to breed, as only one breeding pair is allowed per territory (patch).

Observation At each time step (each week), we record the population size (by gender and total population) and the population age distribution. The number of offspring produced from each birth is recorded after each birth. Each year the genotype frequencies are recorded on week 25 (the last week new litters for the year will be birthed). We also record the per capita growth rate and the yearly per capita growth rate of the fox population. The population size for the growth rates is also taken at week 25 of each year. Observations were made over 100 simulations (per parameter set) in order to see how the stochasticity of the model affects results.

\subsection{Details}

Initialization The model is initialized with the population of foxes defined by the user. Females are placed randomly in territories, and males of breeding age are placed in territories with females of breeding age, if any exist. The user also defines the natural death rate $(\mu)$, and the percent decrease in fecundity due to a homozygous recessive and heterozygous genotypes $(s$ and $h$ ). The baseline successful reproductive rate $(r)$ is 0.61 , and the maximum successful reproductive age $(a)$ is 4 years (208 weeks) [9].

Submodels The various submodels of the ABM are described here in detail.

DisPeRsal When an unmated individual reaches reproductive age (40 weeks), it will disperse in order to establish its own territory and find a mate. An unmated individual of reproductive age will move once within each time step (if there are available patches) until it finds a mate, at which point it will remain on its current patch and establish a territory. This remains the territory of the mated pair until one of the individuals in the pair dies. It is assumed foxes can sense the age and sex of foxes within a $2.96 \mathrm{~km}$ radius (4 patch lengths) of themselves, and will define patches as either possible destinations or preferred destinations. A possible destination is any patch without another fox of the same sex of reproductive age already occupying that patch. A preferred destination is a patch that contains at least one fox of the opposite sex of reproductive age who currently does not have a mate. If preferred destinations exist, a fox will randomly move to one of the preferred destinations. If no preferred destinations exist, a dispersing fox will move to a possible destination if it exists, but otherwise it will remain in its current patch.

Set Alleles Each individual has a genotype containing two alleles. Each allele can be either dominant or recessive. When individuals are born, one of their alleles is determined by randomly choosing one of its mother's alleles, while the second is determined by randomly choosing one of its father's alleles. Individuals with two dominant alleles have a homozygous dominant genotype. Individuals with two recessive alleles have a homozygous recessive genotype. Individuals with one dominant and one recessive allele have a heterozygous genotype.

Breeding Season The breeding season is from weeks 7 to 11, where week 1 is the first week of January [20]. A mated pair will attempt to procreate once every time step (each week) during the breeding season, unless the female in the mated pair is already pregnant. The chance of successful reproduction per attempt is $r$ if the female in the mated pair is homozygous dominant, $r(1-s)$ is the female is homozygous recessive, and $r(1-h s)$ is the female is heterozygous. 
Gestation Once a mating is successful, the gestation week of the female is set to one and increases by one with each week that passes. When the gestation week reaches 7 , the female will give birth [20].

BIRTH After the gestation week of a female reaches 7 , the female gives birth. The number of offspring born is randomly chosen with a $55.8 \%$ chance of producing 1 offspring, a $35.6 \%$ chance of producing 2 offspring, and a $8.6 \%$ change of producing 3 offspring [9]. The age of each offspring is set to zero, the genotype of each offspring is determined by the Set Alleles submodel, and the sex of each offspring is chosen randomly with a $50 \%$ of being female.

DEATH At each time step, each individual has a probability $\mu$ of dying. Death removes mating links, allowing for those with deceased partners to disperse and mate again.

\section{Results}

In the following sets of results we start simulations with either 20, 30, or 50 foxes each with a 1:1 sex ratio. These initial population sizes where chosen to demonstrate population sizes which results in low, medium, and high probabilities of extinction when $s=h=0$. For each initial population size, $100 \mathrm{ABM}$ simulations (with $s=h=0$ ) were run stopping either at 150 years or when the population went extinct, whichever came sooner. In the simulations beginning with 20 foxes, 91\% resulted in extinction before 150 years. In the

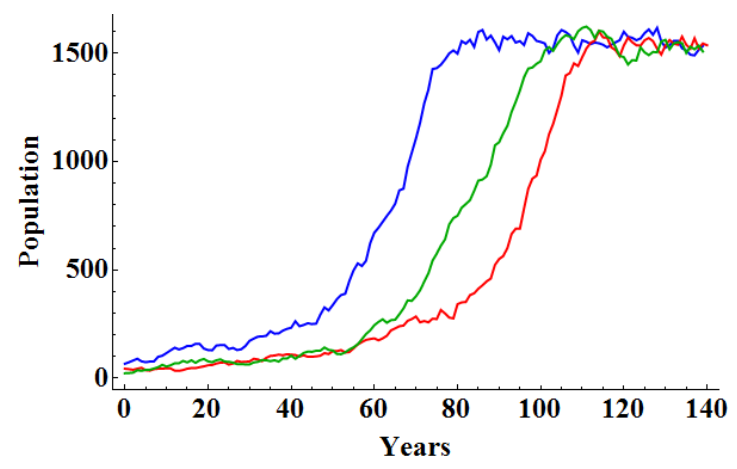

Figure 2: This graph shows the growth in the fox population over time when the initial populations are at 20 individuals (green), 30 individuals (red), and 50 individuals (blue).

simulations beginning with 30 foxes, $69 \%$ resulted in extinction before 150 years. Lastly, in the simulations beginning with 50 foxes, $28 \%$ of the simulations resulted in extinction before 150 years. In the simulations when the population did not go extinct before 100 years, but instead increased overtime, the population appeared to grow logistically eventually reaching a carrying capacity of around 1500 foxes.

Figure 2 shows the population size over time $\left(P_{t}\right)$ in three different simulations where the population eventually reached carrying capacity $(\approx 1500)$ starting with population sizes of $P_{0}=20$ (green), $P_{0}=30$ (red), and $P_{0}=50$ (blue). Note, when the initial populations are at lower values, 20 and 30 foxes (green and red curves, respectively), it takes the population longer to reach carrying capacity than it does when the initial population is higher (50 individuals). Due to the model's stochasticity, it actually takes the simulation starting with 20 foxes longer to reach carrying capacity than the population starting with 30 individuals. However, over many simulations, on average it will take populations starting with 20 foxes longer to reach carrying capacity than populations starting with 30 foxes.

To determine the effect of initial population size on the growth rate of the population, we ran 100 simulations (with $s=h=0$ ) for initial population sizes of 20, 30, and 50 foxes each with 1:1 sex ratios in the initial population. Each year at week 25 we recorded the 


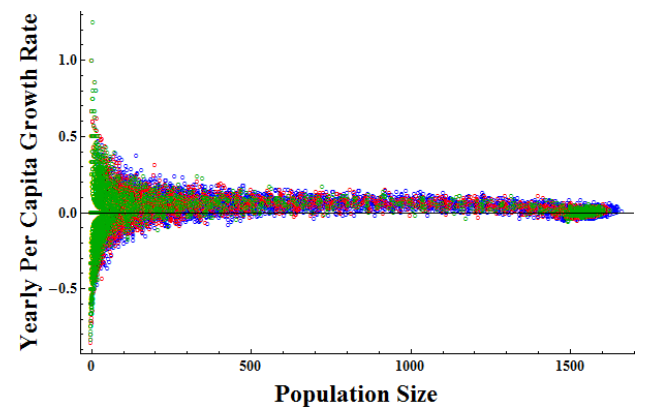

(a) Yearly per capita growth rate plotted against population size.

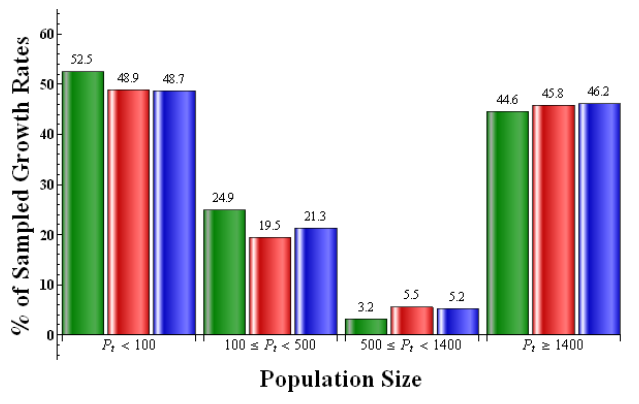

(b) Percent of sampled yearly per capita growth rates which are negative.

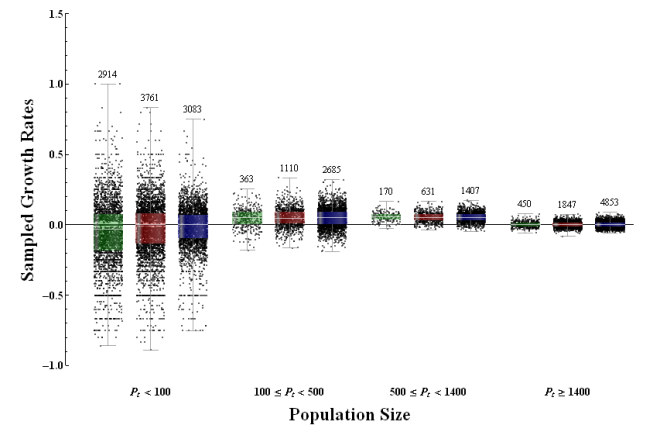

(c) Range of sampled yearly per capita growth rates. Shaded regions indicate the interquartile range, white lines show the median value, and the numbers above each column indicate the number of sample points in that column.

Figure 3: Initial population sizes are 20 foxes (green), 30 foxes (red), and 50 foxes (blue), with $s=0$ and $h=0$. All population size samples taken at week 25 each year of the simulation. Simulations lasted 150 years or until the population went extinct, whichever came first.

population size and calculated the yearly per capita growth rate as

$$
\frac{P_{t}-P_{t-1}}{P_{t-1}}
$$

where $P_{t}$ is the population size in year $t$. Then sample points $\left(P_{t}, \frac{P_{t}-P_{t-1}}{P_{t-1}}\right)$ for each yearly sample for each of the 100 simulations for each initial population size were plotted (see Figure 3a where initial populations sizes are $P_{0}=20$ (green), $P_{0}=30$ (red), and $P_{0}=50$ (blue)). Note that when the population size is low $\left(P_{t}<100\right.$ foxes), many of the sampled yearly per capita growth rates are negative and the range of sampled yearly per capita growth rates is large. However, as the population size increases we see both the range of sampled yearly per capita growth rates and the proportion of sampled yearly per capita growth rates which are negative decrease until the population size nears carrying capacity $\left(P_{t} \geq 1400\right)$. Notice that for population sizes $500 \leq P_{t} \leq 1400$ the sampled yearly per capita growth rates are highly likely to be positive showing that populations in this size range are most likely growing from year to year.

Figure 3b highlights this trend showing the proportion of the yearly per capita growth rates which are negative for four different population size categories: $P_{t}<100,100 \leq P_{t}<$ $500,500 \leq P_{t}<1400$, and $P_{t} \geq 1400$. The bars are colored according to initial population size: 20 foxes (green), 30 foxes (red), and 50 foxes (blue). When comparing the proportion of negative yearly per capita growth rates of the initial population sizes and $P_{t}<100$, a two-sample proportion test showed that a statistically significant difference occurred with the increase of the initial population from 20 to 30 foxes $(p=0.003)$, but not when the 
initial population changed from 30 to 50 foxes $(p=0.88)$. However, the difference in the proportions of negative yearly per capita growth rates between small populations $\left(P_{t}<100\right)$ and larger populations $\left(100 \leq P_{t}<500\right)$ was statistically significant for all initial population sizes $(p<0.0001)$.

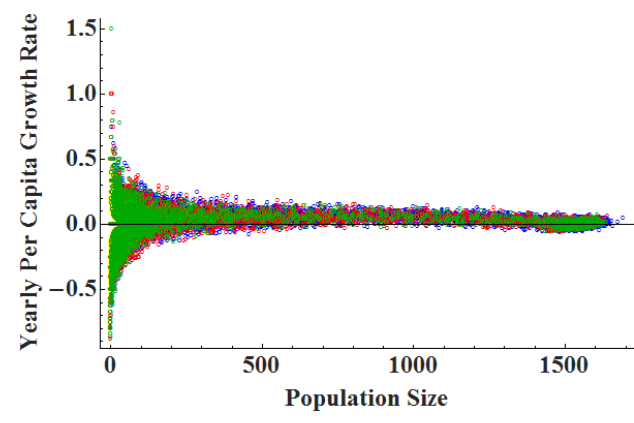

(a) Yearly per capita growth rate plotted against population size.

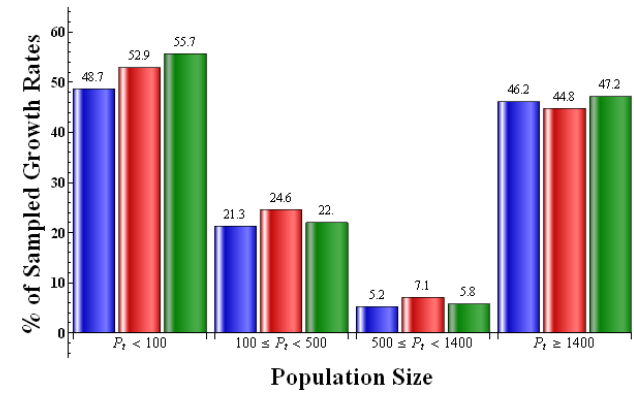

(b) Percent of sampled yearly per capita growth rates which are negative.

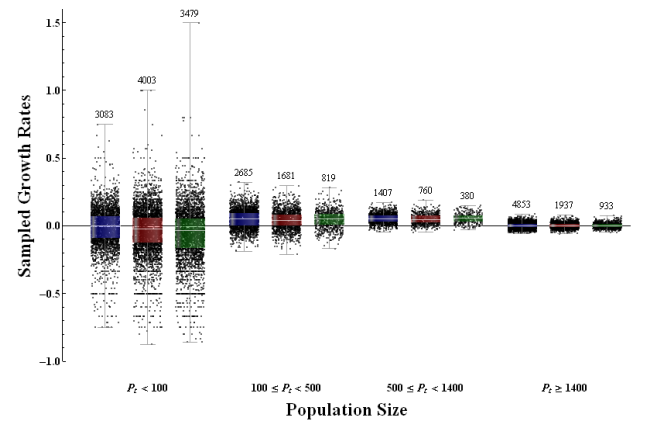

(c) Range of sampled yearly per capita growth rates. Shaded regions indicate the interquartile range, white lines show the median value, and the numbers above each column indicate the number of sample points in that column.

Figure 4: Initial population size is 50 foxes, with $s=0$ (blue), $s=0.5$ (red), and $s=0.9$ (green), and $h=0$. All population size samples taken at week 25 each year of the simulation. Simulations lasted 150 years or until the population went extinct, whichever came first.

While Figure 3b shows the probability of negative yearly growth rates, Figure 3c shows the range of yearly per capita growth rates, and the number of samples within each of the four population size categories for $P_{0}=20$ (green), $P_{0}=30$ (red), and $P_{0}=50$ (blue). Note the colored shaded regions show the interquartile range of the sampled growth rates, the white lines indicate the median value, and the number above each column gives the number of sample points within that column. Recall for simulations with $P_{0}=20,91 \%$ resulted in extinction by 150 years. Thus, there are fewer sample points for larger populations sizes $P_{t} \geq 100$, than for simulations with $P_{0}=30$ and $P_{0}=50$.

It is hypothesized that if a genotype reduces fitness, it can negatively affect the per capita growth rate of the population. In our model a detrimental recessive allele reduces individual reproductive success. To determine what effect the presence of the recessive allele has on the population dynamics and yearly per capita growth rates, we ran 100 simulations for each of the following parameter scenarios: (1) $s=0, h=0,(2) s=0.5, h=0,(3) s=0.5$, $h=0.5$, (4) $s=0.5, h=1$, (5) $s=0.9, h=0$. Each scenario used an initial population of 50 foxes and was simulated for 150 years or until the population went extinct, whichever came first.

The graphs in Figure 4 are similar to those in Figure 3 except the colors correspond to parameter scenarios (1) $s=0, h=0$ (blue), (2) $s=0.5, h=0$ (red), and (5) $s=0.9$, 
$h=0$ (green). Figure 4a shows trends similar to those in Figure 3a. Figure 4b shows the percentage of sampled yearly per capita growth rates which are negative for four different population size categories: $P_{t}<100,100 \leq P_{t}<500,500 \leq P_{t}<1400$, and $P_{t} \geq 1400$. Notice for low populations sizes $\left(P_{t}<100\right)$ increasing the value of $s$ correlates to increasing the probability having a negative yearly growth rate, however this trend does not exist in the larger population sizes $\left(P_{t} \geq 100\right)$. A two-sample proportion test showed a statistically significant difference in the proportion of negative yearly per capita growth rates in small populations $\left(P_{t}<100\right)$ when increasing $s$ from 0 to $0.5(p<0.0001)$, and for increasing $s$ from 0.5 to 0.9 , though with less confidence $(p=0.02)$. Additionally, a two-sample proportion test was used to determine that the difference in the proportions of negative yearly per capita growth rates between small populations $\left(P_{t}<100\right)$ and larger populations $\left(100 \leq P_{t}<500\right)$ for all simulated values of $s$ were statistically significant $(p<0.0001)$.

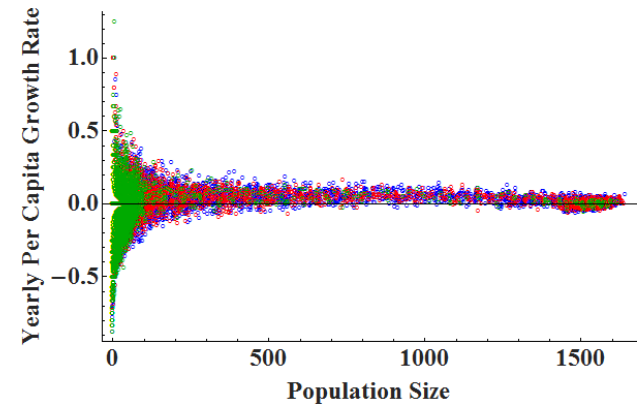

(a) Yearly per capita growth rate plotted against population size.

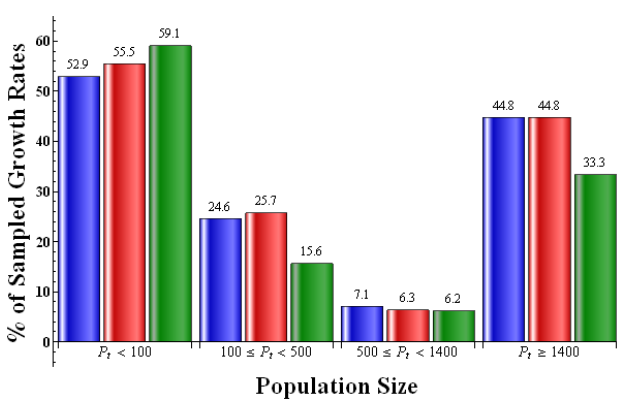

(b) Percent of sampled yearly per capita growth rates which are negative.

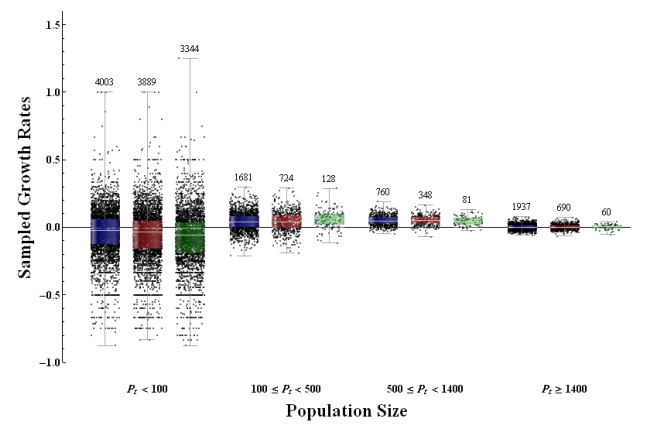

(c) Range of sampled yearly per capita growth rates. Shaded regions indicate the interquartile range, white lines show the median value, and the numbers above each column indicate the number of sample points in that column.

Figure 5: Initial population size is 50 foxes with $s=0.5, h=0$ (blue), $h=0.5$ (red), and $h=1$ (green). All population size samples taken at week 25 each year of the simulation. Simulations lasted 150 years or until the population went extinct, whichever came first.

Figure $4 \mathrm{c}$ is a density plot showing the range of yearly per capita growth rates over all simulations with parameter sets (1) $s=0, h=0$ (blue), (2) $s=0.5, h=0$ (red), and (5) $s=0.9, h=0$ (green). Note changing the value of $s$ has little effect on the range of yearly per capita growth rates, except for small population sizes $\left(P_{t}<100\right)$. However, the number of points contributing to each the population size category decreases as $s$ is increased. This is due to the fact that when $s=0$ a high number of simulations reach carrying capacity $\left(P_{t} \geq 1400\right)$. As $s$ increases, the number of simulations where the population reaches carrying capacity declines.

The graphs in Figure 5 are similar to those in Figure 4 except the colors correspond to parameter scenarios (2) $s=0.5, h=0$ (blue), (3) $s=0.5, h=0.5$ (red), and (4) $s=0.5$, 
$h=1$ (green). Notice Figure 5a shows trends similar to those in Figures 3a and 4a, except that the green data $(s=0.5$ and $h=1)$ is virtually non-existent for population sizes over 100 . This is because of the 100 simulations with $s=0.5$ and $h=1$, only two simulations had a population which grew to reach carrying capacity. This can be seen more directly in Figure 5c which shows only 60 data points for the $P_{t} \geq 1400$ category when $h=1$ (green). These 60 data points correspond to samples over 6 years in one simulation and over 54 years in the second simulation.

Figure 5b shows the percentage of sampled yearly per capita growth rates which are negative for the four different population size categories. Notice, similar to Figure 4b, for low population sizes $\left(P_{t}<100\right)$ increasing the value of $h$ correlates to increasing the probability of having a negative yearly growth rate, however this trend does not exist for larger population sizes $\left(P_{t} \geq 100\right)$. A two-sample proportion test was used to determine that the difference in the proportions of negative yearly per capita growth rates between small populations $\left(P_{t}<100\right)$ and larger populations $\left(100 \leq P_{t}<500\right)$ for all simulated values of $h$ were statistically significant $(p<0.0001)$. Additionally, a two-sample proportion test showed that there was a statistically significant difference in the proportion of the negative yearly per capita growth rates in small populations $\left(P_{t}<100\right)$ when increasing $h$ from 0 to $0.5(p=0.02)$ and from 0.5 to $1(p=0.002)$.

In Figures 3b, 4b, and 5b we see evidence of the Allee effect in the increased probability of having a negative yearly per capita growth rate at low population sizes $\left(P_{t}<100\right)$. Furthermore, when we compare Figure $5 \mathrm{~b}$ to Figure $4 \mathrm{~b}$ (in which the value of $s$ was varied), we see that non-zero values of $h$ appear to cause a higher probability of a having a negative growth rate in small populations $\left(P_{t}<100\right)$ than $h=0$ with non-zero values of $s$. In Table 1 we examine percentage of samples of yearly per capita growth rates which are negative for $P_{t}<100$ for different initial population sizes and different genetic effects on individual fitness (i.e. reduction in reproductive success). Note that for each initial population size, the percentage of samples of yearly per capita growth rates which are negative increase as we increase the number of genotypes which would experience reduced fitness from no genotypes causing a reduction in reproductive success $(s=0$ and $h=0)$, to only homozygous recessive genotypes causing reduced reproductive success $(s=0.5$ and $h=0)$, and finally to homozygous recessive and heterozygous genotypes causing reduced reproductive success $(s=0.5$ and $h=0.5)$. A two-sample proportion test confirmed that for initial population sizes of 30 and 50 foxes the percentage of negative yearly per capita growth rates found in Table 1 showed a statistically significant increase when more genotypes cause diminished reproductive success. Specifically, when only homozygous recessive genotypes cause reduced reproductive success, both initial population sizes showed a statistically significant increase in negative yearly per capita growth rates $\left(P_{0}=30, p=0.01 ; P_{0}=50, p<0.0001\right)$ when compared to no genotypes causing reduced reproductive success. Additionally, when both homozygous recessive and heterozygous genotypes cause reduced reproductive success, both initial populations showed statistically significant increases in negative yearly per capita

Table 1: Percentage of samples with negative yearly per capita growth rates for $P_{t}<100$ with no genotypes cause a reduction in reproductive success $(s=0$ and $h=0)$, only homozygous recessive genotypes causing reduced reproductive success $(s=0.5$ and $h=0)$, and homozygous recessive and heterozygous genotypes causing reduced reproductive success $(s=0.5$ and $h=0.5)$.

\begin{tabular}{lccc}
\hline & $P_{0}=20$ & $P_{0}=30$ & $P_{0}=50$ \\
\hline No Genotypes & 52.5 & 48.9 & 48.7 \\
Homozygous Recessive Only & 53.5 & 52.0 & 52.9 \\
Homozygous Recessive + Heterozygous & 55.1 & 56.6 & 55.5 \\
\hline
\end{tabular}


growth rates $\left(P_{0}=30, p<0.0001 ; P_{0}=50, p=0.02\right)$ when compared to only homozygous recessive genotypes causing reduced reproductive success. For simulations beginning with 20 foxes, a two-sample proportion test showed the percentage of yearly per capita growth rates in Table 1 showed no statistically significant increase when more genotypes cause diminished reproductive success. Specifically, there was no significant increase in the percentage of yearly per capita growth rates when only homozygous recessive genotypes caused reduced reproductive success $(p=0.46)$ when compared to no genotypes causing reduced reproductive success, and when both homozygous recessive and heterozygous genotypes caused reduced reproductive success $(p=0.28)$ when compare to only homozygous recessive genotypes causing reduced reproductive success, and $(p=0.07)$ when compared to no genotypes causing reduced reproductive success.

Table 2: Mean of negative yearly per capita growth rates for $P_{t}<100$ with no genotypes cause a reduction in reproductive success $(s=0$ and $h=0)$, only homozygous recessive genotypes causing reduced reproductive success $(s=0.5$ and $h=0)$, and homozygous recessive and heterozygous genotypes causing reduced reproductive success $(s=0.5$ and $h=0.5)$.

\begin{tabular}{lrrr}
\hline & $P_{0}=20$ & $P_{0}=30$ & $P_{0}=50$ \\
\hline No Genotypes & -0.223 & -0.180 & -0.129 \\
Homozygous Recessive Only & -0.226 & -0.215 & -0.160 \\
Homozygous Recessive + Heterozygous & -0.250 & -0.237 & -0.182 \\
\hline
\end{tabular}

Table 2 displays the average yearly per capita growth rate for all negative rates and for each initial population size. An increase in the magnitude of negative yearly per capita growth rates corresponds to a stronger Allee effect, as graphically displayed in Figure 1. After performing a series of two-sample $t$-tests, all mean negative yearly per capita growth rate reductions found in Table 2 are significant for each initial population size $(p<0.0001)$, with the exception of the decrease from no genotypes effected to the homozygous recessive genotype for $P_{0}=20(p=0.66)$. For small initial population sizes $\left(P_{0}=20\right)$, a lesser change in effect is expected due to the large percentage of populations that quickly become extinct.

\section{Conclusions}

The purpose of this model was to determine whether the island fox population displays the Allee effect, in which low population density is directly correlated to low fitness levels in either the population (demographic) or individuals (component), as well as to see whether the presence of a detrimental allele could induce or exacerbate the Allee effect. When we left the population unaffected by a detrimental recessive allele $(s=0)$, the population exhibited the expected logistic growth with an Allee effect a low population levels as evident by the increased probability of having a negative yearly per capita growth rate at low population sizes (less than 100 individuals). When both the homozygous recessive and heterozygous genotypes reduce reproductive success, the probability of having a negative yearly per capita growth rate at low population sizes increases. When only the homozygous recessive genotype reduced reproductive success, there was evidence that the probability of having a negative yearly per capita growth rate at low population sizes increased; thus, this alone did not exacerbate the Allee effect. However, simulations where only the homozygous recessive genotype reduced reproductive success were more likely to go extinct before 100 years, and the likelihood increased when heterozygous genotypes also reduced reproductive success.

The results from this agent-based model can be compared to those from both discrete and continuous-time models. In [6], Boukal \& Berec show the typical curves of the per 
capita growth rate as a function of population size for both discrete-time and continuoustime singles-species models which incorporate an Allee effect. We show a similar graph in Figure 1. The Allee effect in these graphs is shown as negative or small positive per capita growth rates for small population sizes. The per capita growth rate increases with population size until the population size nears carrying capacity. When we compare the results from our model with the trends presented by Boukal \& Berec, we see that our model does match this pattern (see Figures 3a, 4a, and 5a). However, since our model is stochastic and individual-based, the yearly per capita growth rate does have some probability of being positive, even at low population sizes. Thus, in our model the Allee effect manifests itself as a higher probability of having a negative year per capita growth rate at small population sizes. In population sizes between 100 and 1400, there is a much higher probability (roughly $75-97 \%$ ) that the year per capita growth rate is positive. Thus, the population is more likely to continue growing to carrying capacity if it can a size greater than 100 foxes. The median yearly per capita growth rate drops to nearly zero when the population size nears carrying capacity.

This research demonstrates that an Allee effect could have hindered the re-establishment of the island fox population on Santa Cruz island after their precipitous decline in the early 1990s. Additionally, if a mutation was introduced into the genome of the population at some point preceding the population decline, this could have contributed to the extreme decline in the population once predation, competition, and disease worked in combination to initially lower the population size. More importantly, our research shows even if there is a detrimental allele present, it is possible to get the fox population back to carrying capacity if the population can be brought up to around 100 foxes (for example, through augmentation or captive breeding), after which it is more likely that yearly per capita growth rates will be positive.

\section{Acknowledgements}

This work was supported in part by a faculty development grant from the Association of Southern Colleges awarded to ENB and AY. Additionally, a the work by SMS was supported by the 2013 Rhodes Summer Biomathematics Fellowship and by a Rhodes Student Research Assistantship (Fall 2013). Lastly, all the authors would like to thank Jim Crowder, Huda Qureshi, and Allison Russel to their input and feedback on this project.

\section{References}

[1] W. C. Allee. Integration of problems concerning protozoan populations with those of general biology. American Naturalist, 75:473-487, 1941.

[2] E. Angulo, G. W. Roemer, L. Berec, J. Gascoigne, and F. Courchamp. Double allee effects and extinction in the island fox. Conservation Biology, 21(4):1082-1091, 2007.

[3] E. N. Bodine, L. J. Gross, and S. Lenhart. Optimal control applied to a model for species augmentation. Mathematical Biosciences and Engineering, 4(5):669-680, 2008.

[4] E. N. Bodine, L. J. Gross, and S. Lenhart. Order of events matter: comparing discrete model for optimal control of species augmentation. Journal of Biological Dynamics, 6(Supplemental 2):31-49, 2012.

[5] C. Boser. Santa Cruz Island Fox Recovery Program. Technical report, State of California Natural Resources Agency Department of Fish and Game Wildlife Branch, 20112012 . 
[6] D. S. Boukal and L. Berec. Single-species models of the allee effect: Extinction boundaries, sex ratios, and mate encounters. Journal of Theoretical Biology, 218:375-394, 2002.

[7] H. O. Clark. Species at risk: Golden eagle predation on arid-land foxes. Endangered Species Update: Science, Policy, \&3 Emerging Issues, 26(1 \& 2):10-14, 2009.

[8] D. L. Clifford, J. A. K. Mazet, E. J. Dubovi, D. K. Garcelon, T. J. Coonan, P. A. Conrad, and L. Munson. Pathogen exposure in endangered island fox (Urocyon littoralis) populations: Implications for conservation management. Biological Conservation, 131:230$243,2006$.

[9] T. J. Coonan. Recovery strategy for island foxes on the northern channel islands. Technical report, National Park Service: Channel Islands National Park, 2003.

[10] F. Courchamp, B. Grenfell, and T. Clutton-Brock. Population dynamics of obligate cooperators. Proceedings of the Royal Society of London B, 266:557-563, 1999.

[11] F. Courchamp, R. Woodroffe, and G. Roemer. Removing protected populations to save endangered species. Science, 302:1532, 2003.

[12] D. L. DeAngelis and W. M. Mooij. Individual-based modeling of ecological and evolutionary processes. Annual Review of Ecology, Evolution, and Systemics, 36:147-168, 2005 .

[13] V. Grimm, U. Berger, D. L. DeAngelis, J. G. Polhill, J. Giske, and S. F. Railsback. The ODD protocol: A review and first update. Ecological Modelling, 221:2760-2768, 2010.

[14] K. L. Jones, D. H. Van Vuren, and K. R. Crooks. Sudden increase in a rare endemic carnivore: ecology of the island spotted skunk. Journal of Mammology, 89(1):75-86, 2008.

[15] A. M. Kramer, B. Dennis, A. M. Liebhold, and J. M. Drake. The evidence of Allee effects. Population Ecology, 51:341-354, 2009.

[16] R. López-Ruiz and D. Fournier-Prunaret. Indirect Allee effect, bistability and chaotic oscillations in a predator-prey discrete model of logistic type. Chaos, Solitons \& Fractals, 24:85-101, 2005.

[17] S. F. Railsback and V. Grimm. Individual-Based Modeling and Ecology. Princeton Series in Theoretical and Computational Biology. Princeton University Press, Princeton, NJ, 2005 .

[18] S. F. Railsback and V. Grimm. Agent-Based and Individual-Based Modeling: A Practical Introduction. Princeton University Press, 2011.

[19] G. W. Roemer, C. J. Donlan, and F. Courchamp. Golden eagles, feral pigs, and insular carnivores: how exotic speices turn native predators into prey. Proceedings of the National Academy of Sciences, 99(2):791-796, 2002.

[20] J. Sanchez and B. Hudgens. Spatial ecology of the island fox. Technical report, Department of Defense Legacy Resource Management Program, 2011.

[21] S. Zhou, Y. Liu, and G. Wang. The stability of predator-prey systems subject to the allee effects. Theoretical Population Biology, 67:23-31, 2005. 